\title{
Literature Watch
}

\author{
Reviewed by Gerhard Litscher, MSc, PhD, MDsc
}

\section{The efficacy of acupuncture in postoperative sleep quality: a literature review.}

Song B, Luo M, Zhu J. Sleep Breath. 2020;September 19:e-pub.

G ENERAL ANESTHESIA CAN AFFECT the quality of postoperative sleep, especially after surgery on elderly patients. This decline of postoperative sleep quality could have harmful effects on postoperative recovery of such patients. This review, by authors from different anesthesiologic departments in Chinese medical centers, summarized the efficacy and potential mechanism of acupuncture on postoperative sleep quality. The researchers reviewed the effect of general anesthesia on the human circadian sleep rhythm. They also offer evidence on impairments caused by decreased postoperative sleep quality, emphasizing the mechanism of acupuncture that can alleviate factors affecting sleep quality after general anesthesia.

Acupuncture has been helpful for improving sleep quality and alleviating postoperative complications affecting postoperative sleep quality after general anesthesia. Acupuncture at different acupoints could improve the body's neurotransmitter levels effectively and regulate biologic clock genes through various mechanisms. Acupuncture could thus improve postoperative sleep quality. Large-scale multicenter trials are needed to support these findings.

\section{Moxibustion enhances chemotherapy of breast cancer by affecting tumor microenvironment.}

Xue N, Fu X, Zhu Y, Da N, Zhang J. Cancer Manag Res. 2020; 12:8015-8022.

$\mathbf{C}$ HEMOTHERAPEUTIC DRUGS often cause obvious toxicity and side-effects. Moxibustion can improve the immunity of patients who have cancer, enhance their cellular immunity, and reduce the toxicity and adverse effects of radiotherapy and chemotherapy. Researchers from the south of China evaluated the efficacy of moxibustion + paclitaxel on breast cancer. A breast- cancer mouse model was established. Hematoxylin and eosin staining were used to analyze tumor necrosis in mouse tumors. Immunohistochemistry, Western blot, and qPCR [polymerase chain reaction] were used to detect expression of CD34, hypoxia-inducible factor-1 $\alpha(H I F-1 \alpha)$, vascular endothelial growth factorA (VEGFA), programmed death-1 (PD-1), and programmed death-1 ligand $(P D-L 1)$ in tumor tissues.

Moxibustion + paclitaxel inhibited weight loss significantly in the breast-cancer-burdened mice and increased their survival rate. This combination treatment also: (1) increased the number of white blood cells in the mice; (2) increased their thymus and spleen indices; and (3) enhanced the animals' immune function by upregulating interferon- $\gamma$ and interleukin-2 and downregulating interleukin-10 and transforming growth factor- $\beta 1$.

Notably, moxibustion + paclitaxel inhibited angiogenesis of tumors through downregulation of CD34, HIF-1 $\alpha$, and $V E G F A$, and overcame the immunosuppressive microenvironment by inhibiting the $P D-1 / P D-L 1$ signaling pathway. Moxibustion improves the body's immune function and enhances the efficacy of chemotherapy by overcoming the immunosuppressive microenvironment.

\section{Acupuncture for lateral epicondylitis: a PRISMA- compliant protocol for a systematic review and meta-analysis of randomized controlled trials.}

Kim HN, Goo B, Nam SS. Medicine (Baltimore). 2020;99(37): e22008.

A CUPUNCTURE HAS BEEN USED WIDELY to relieve pain associated with musculoskeletal disorders, such as lateral epicondylitis. Although the effect of acupuncture on pain has been demonstrated in previous reviews, it is still debated. The goal of this study was to evaluate the efficacy of acupuncture for treating lateral epicondylitis and establish this evidence systematically. Nine databases were searched from their inception to May 2020 without language or publication-status restrictions, including 3 English databases 
(MEDLINE ${ }^{\circledR}$ Embase, and the Cochrane Central Register of Controlled Trials), 5 Korean databases (Korean Medical Database, KoreaMed, Korean Studies Information Service System, Research Information Service System, and the Oriental Medicine Advanced Searching Integrated System), and 1 Chinese database (the China Knowledge Network Database).

Only randomized controlled trials were included. Pain intensity was considered as the primary outcome. Secondary outcomes included grip strength, total effective rate, and adverse events. Two independent researchers performed the study selection, data extraction, and quality assessment. The methodological quality of the identified studies were assessed, using the Cochrane Collaboration's risk-of-bias tool. In the meta-analysis, continuous data were expressed as mean and 95\% confidence interval (CI), and dichotomous data were expressed as risk ratio and $95 \%$ CI.

The results of this study will be submitted to a peerreviewed journal for publication. The results could provide evidence for acupuncture's effectiveness for addressing lateral epicondylitis.

\section{Acupuncture as a treatment within integrative health for palliative care: a brief narrative review of evidence and recommendations.}

Birch S, Bovey M, Alraek T, Robinson N, Kim TH, Lee MS. J Altern Complement Med. 2020;26(9):784-791.

A CUPUNCTURE IS ONE of the fastest-developing evidence bases in complementary medicine and is one of the leading therapies included as part of integrative health care. This narrative review has 2 separate parts: (1) an evaluation of the current evidence status of reviews of acupuncture and (2) an examination, with examples, of recommendations for using acupuncture per treatment guidelines from health care experts and public-health organizations that recommend acupuncture as a viable treatment for patients in palliative care.

Electronic searches were performed in PubMed using the terms acupuncture + palliative, and adding safety to find review articles documenting safety and evidence of effectiveness of acupuncture for treating symptoms experienced by patients in palliative care. Treatment guidelines that recommend use of acupuncture for symptom control in palliative care were found by searching through a database currently under construction by the lead author of this review.

Acupuncture had emerging evidence for 17 indications in palliative care. There were examples of publications recommending acupuncture for treating symptoms for patients in palliative care from government, public-health, oncology, and medical-expert sources. The most publications were in oncology but other situations were included, such as pediatric care. While the evidence for using acu- puncture to treat symptoms patients undergoing palliative care is relatively weak, the evidence base is growing. Experts from around the world are increasingly recommending acupuncture to treat symptoms of patients in palliative care.

Given that acupuncture is a safe, nonpharmacologic treatment with small clinically significant effects, these recommended uses appear to be pragmatic efforts to bridge the gap of treatment options available to these patients.

\section{Machine learning in neuroimaging: a new ap- proach to understand acupuncture for neuroplas- ticity.}

Yin T, Ma P, Tian Z, Xie K, He Z, Sun R, Zeng F. Neural Plast. 2020;2020:8871712.

\section{$\mathbf{T}$} HE EFFECTS OF ACUPUNCTURE facilitating neural plasticity for treating diseases have been identified by clinical and experimental studies. During the last 2 decades, neuroimaging techniques in acupuncture research have provided visual evidence for acupuncture promoting neuroplasticity. Recently, integration of machine learning and neuroimaging techniques has becomes focus in neuroscience and is bringing a new and promising approach to understanding the use of acupuncture to effect neuroplasticity at the individual level.

This review was prepared to provide an overview of this rapidly growing field by introducing the commonly used machine learning algorithms in neuroimaging studies briefly and by analyzing the characteristics of the acupuncture studies based on machine learning and neuroimaging, in order to provide references for future research.

\section{Acupuncture ameliorated vasomotor symptoms during menopausal transition: single-blind, placebo-controlled, randomized trial to test treat- ment efficacy.}

Soares JM Jr, Branco-de-Luca AC, da Fonseca AM, et al. Menopause. 2020; September 7:e-pub ahead of print.

$\mathbf{T}$ HIS GOAL OF THIS STUDY was to evaluate the effects of acupuncture on women with vasomotor symptoms during the menopausal transition. The Kupperman-Blatt Menopausal Index was used as a study tool. This was a crossover, singleblinded, sham-controlled trial with 100 women randomly divided into 2 groups of 50 participants each: G1 and G2. During the first 24 weeks of the study, the G1 women received verum acupuncture and the $\mathrm{G} 2$ women were given sham acupuncture. The crossover was then applied so that the G1 participants were 
given sham acupuncture and the G2 participants received verum acupuncture for 24 more weeks.

The mean score of hot flashes of the group who first experienced verum acupuncture (G1) was statistically higher than that of the group who started with sham acupuncture (G2; $P=0.020)$. In addition, both groups had similar mean scores in the middle of the study (both were receiving verum acupuncture at that timepoint). During the last 6 months of the study, after the crossover, the values of the G2 (verum acupuncture) group were lower than those of G1 (sham acupuncture) group.

Verum acupuncture treatment could mitigate hot flashes and other climacteric symptoms during the menopausal transition.

\section{Acupuncture versus various control treatments in the treatment of migraine: a review of randomized controlled trials from the past $\mathbf{1 0}$ years.}

Ni X, Dong L, Tian T, Liu L, Li X, Li F, Zhao L. J Pain Res. 2020;13:2033-2064.

$\mathbf{M}$ IGRAINE IS A RECURRENT HEADACHE of moderate-tosevere intensity that affects quality of life seriously. Recent clinical trials have confirmed that acupuncture is effective for treating migraine. Researchers from Chengdu reviewed the effectiveness of acupuncture for treating migraine by comparing treatment and various control groups according to newly published guidelines for systematic reviews.

The following databases were searched for relevant articles published from January 1, 2010 to December 31, 2019: Embase; PubMed; Medline ${ }^{\circledR}$; the Cochrane Library; and 4 Chinese databases. The current review included randomized controlled trials of acupuncture either as a single treatment or as an ad- junctive treatment for migraine. Two researchers conducted the study selection, data extraction, and quality-assessment processes independently. Disagreements between the reviewers were resolved through discussion and data reanalysis.

Quality of each included study was evaluated using the Cochrane Collaboration risk-of-bias assessment method and the STandards for Reporting Interventions in Controlled Trials of Acupuncture (STRICTA) checklist. Forty-nine studies were analyzed and ranked based on these standards. The analysis revealed that acupuncture reduced headache frequency, compared to no treatment (mean difference [MD] $=-1.80 ; P<0.00001 ; 95 \%$ confidence interval $[\mathrm{CI}]$ : -2.34 to -1.26$)$ and Western Medicine $(\mathrm{MD}=-1.75$; $P=0.003$; 95\% CI: -2.91 to -0.58$)$. Headache frequency did not differ significantly between patients who received real acupuncture versus those who received sham acupuncture ( $\mathrm{MD}=-0.64 ; P=0.24 ; 95 \% \mathrm{CI}:-1.70$ to 0.42 ). The reviewers also evaluated current research on using acupuncture to treat migraine, compared with various control treatments.

The researchers stated that the evidence for the effectiveness of acupuncture in controlling migraine is still limited because of the low quality of the published studies.

Address correspondence to: Gerhard Litscher, MSc, PhD, MDsc Research Unit of Biomedical Engineering in Anesthesia and Intensive Care Medicine and TCM Research Center Medical University of Graz. Auenbruggerplatz 39, EG19 8036 Graz Austria

E-mail: gerhard.litscher@medunigraz.at 